\title{
Challenges and Solutions for the Safety of Children in the Community
}

Mitch Blair, MBBS, BSC, MSC, FRCP, FRCPCH, FFPH FRSPH Shermina Sayani, MBBS, BSC, MA, MRCPCH, ,* Elizabeth Wortley, MBBS, BSC, MRCPCH

\author{
Address \\ ${ }^{1}$ Department of Paediatrics and Child Health, Imperial College London, River \\ Island Academic Centre, Northwick Park Campus, Watford Road, Harrow, Middle- \\ sex, HA1 3UJ, UK \\ ${ }^{*}, 2$ Department of Paediatrics and Child Health, River Island Academic Centre for \\ Paediatrics and Child Health, Level 7 Maternity Unit, Northwick Park Hospital, \\ London North West Health Care NHS Trust, Watford Road, Harrow, Middlesex, HA1 \\ 3UJ, UK \\ Email: shermina.sayani@nhs.net
}

Published online: 8 0ctober 2015

(C) Springer International Publishing AG 2015

This article is part of the Topical Collection on Patient Safety

Keywords Child safety - Injury prevention - Safeguarding - Life course - Missed opportunities - Communication . Protocols - Think-box · Reflection - Resilience $\cdot$ Holistic - Advocacy $\cdot$ Quality $\cdot$ Looked after $\cdot$ Health systems . Interagency $\cdot$ Community paediatrics

\section{Opinion statement}

To truly understand the challenges and solutions of the safety of children in our community, we should be willing to embrace its complexity. Child safety itself goes further than some might conceive, encompassing the prevention of all types of injury: physical, emotional and psychological as well as interventions to promote resilience to harm. We believe child safety is a 'call to action' to paediatricians, government and society in general to advocate for children who remain vulnerable and often voiceless. We have chosen to take a narrative approach examining one particular case and have identified different preventable safety issues that occurred throughout her childhood. Acknowledging that early events have an impact later in the life course, we emphasise the importance of prompt early intervention and reflect on 'missed' opportunities for action. In particular, we have tried to use a holistic lens to view the case, exploring issues in the system around the child, in their family, school and the wider environment. In exploring the solutions to these issues, we look at three key areas (the $3 \mathrm{R}$ approach): firstly, effective communication between professionals (relationships) and how this might be practically facilitated at ground level; secondly, how to best ensure protocols 
(roadmaps) are followed wisely within an organisational and human behaviours framework; and finally, by putting the child at the centre (recognition), we can address the multiple issues affecting them and identify factors that build his/her own resilience. Child safety is a complex far reaching public health priority, which requires holistic ways of identifying safety issues, as well as practical solutions that support professionals and empower children and their families.

\section{Introduction}

\section{Child safety}

In order to explore the area of child safety in our community, let us examine how this term is understood and its scope. Child safety most commonly refers to unintentional injuries-one of the leading causes of childhood death and emergency department (ED) attendance in industrialised countries $[1,2]$.

The European Child Safety Alliance (ECSA) suggests a broader definition which includes alcohol, self-harm and suicide, bullying and violence, including, interestingly, child maltreatment [3]. Maltreatment can also occur through the medium of the internet; therefore 'internet safety' has become a priority for government and the police [4].

'Safeguarding' is increasingly used in the UK to indicate a more holistic approach to child safety and includes preventing any impairment to children's health and development, ensuring children grow up to have the best outcomes and implying an 'action orientation' in the definition [5].

A particular form of safeguarding involves institutions or corporations, when their systems can cause inadvertent harm, as described in the seminal report 'To err is human' [6]. This report reminds us that safety goes beyond the individual, to the system or 'macro' level.

Another perspective on child safety is to empower children and their families to cope better with the inherent risks of their childhood-building resilience. Resilience is defined as 'reduced vulnerability to environmental risk experiences, the overcoming of a stress or adversity, or a relatively good outcome despite risk experiences' [7].

Based on the discussion above, we wish to propose our own definition of child safety in the community:

Child Safety is the right of the child or young person to be protected from physical, psychological or social harm through environmental modification, health, education and social system vigilance and the promotion of resilience through education, family and carer nurture, peer support, and professional and community advocacy.
With that definition in mind, how might we identify child safety issues in practice? Patient stories are a way to teach and learn about complexity from multiple perspectives over the whole course of a child's life $[8,9]$. In particular, we can identify which biological, environmental or psychosocial factors may have contributed to later problems [10]. We have decided to use the story of Lucy-a real case of a 17-year-old girl who was seen in the clinic for children in the care of social services. Actual names and details have been changed to protect anonymity. Whilst this example is from our context in the UK, we believe that the lessons learnt are applicable in all societies.

\section{Lucy's story}

Lucy was born in the local maternity unit at 38 weeks gestation in good condition and was discharged home within 48 hours.

Lucy's mother was fleeing from a domestically violent relationship, and she had a history of alcohol abuse and depression, of which the maternity unit were unaware. She had booked late as she had only just moved into the area.

Lucy was seen twice in the emergency department in her early years-age 3 after a fall down the stairs that fractured her arm and age 5 requiring stitches to her head after falling from her bike.

During her primary school years, concerns were raised because of consistently poor attendance and a scruffy appearance.

Social services realised her mother had a chaotic lifestyle and a history of alcohol abuse. The care plan included referrals to parenting and drug and alcohol programmes-though she did not attend. When her social worker changed, a review showed things had improved at home and her case was closed.

Lucy was seen in children's outpatients age 12 with a history of headaches, these did not appear to be significant and she was discharged. 
At age 14, Lucy attended the general (family) practitioner with low mood, who referred to the local Child and Adolescent Mental Health Services (CAMHS) - Lucy did not attend and was discharged.

At age 17, Lucy was seen in a clinic for children in the care of social services. She was living in a youth hostel having approached social services to be 'looked after'. During her medical review, Lucy explained her mother's history and her own role as a carer from the age of 9 .
Whilst maintaining the household, Lucy struggled with the situation-resulting in self-harm and suicidal ideation. She had not made many friends at college and had poor self-esteem. Her boyfriend, who was in financial difficulties, regularly stole money from her. They were not practising safe sex.

After the clinic, Lucy was referred to the CAMHS team but having turned 18, the referral was rejected. Lucy left college and subsequently the care of social services.

\section{Challenges}

The sad truth of Lucy's case is that we only find out about her reality at 17.5 years old, when effectively an adult and about to leave the care system. This was when she had a 'voice' and made it heard. Though she had often been in contact with services, they had not apparently been able to appreciate the full picture. In this next section, we shall review Lucy's case and explore the various points in her life course when a number of safety issues could have been identified and look at the evidence which exists to support interventions.

- Domestic violence or intimate partner violence

Domestic violence or intimate partner violence (IPV) [11] can have a significant impact on child and maternal health outcomes [12]. During pregnancy, frequency of IPV increases [13], becoming a major child safety issue. In Lucy's case, the first 'missed opportunity' was not identifying IPV—so, should we screen for IPV? A National Institute for Health and Care Excellence (NICE) meta-analysis [11] found that universal screening is generally acceptable to clients when used sensitively, with more success when self-administered and addressing other short- and long-term safety concerns. Efficacy of screening requires clear institutional support, training, effective protocols and access to onsite referral systems. Unfortunately, increased screening does not necessarily lead to increased referrals [11], perhaps because of lack of supporting structures for onward referrals. Even if she was referred, there is insufficient evidence to say this would have been successful [14].

- Maternal mental health

Knowing that maternal depression can impact on later attachment [1517] and child development [18], professionals may have been alerted

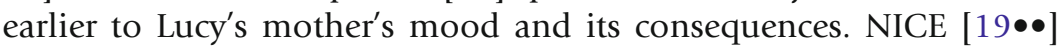
highlights the need to explore mood of a client at the first contact, escalating as appropriate. The Edinburgh Post Natal Depression scale (EPND-S) [20], for example, can be used by health visitors for this purpose. However, as stated before, effectiveness of screening is only as valuable as the system within which it sits. There have been reports of 
inconsistent use of EPND-S perhaps reflecting the limited time and resources available to professionals, to use this tool [21].

- Alcohol misuse

Maternal alcohol use has multiple safety issues for Lucy. Children in such families are more likely to experience adverse childhood events, and there is an association between parental alcohol abuse and depression in their

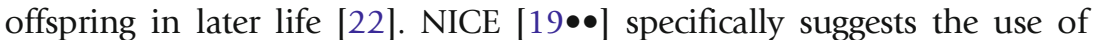
another screening tool such as Alcohol Use Disorder Identification Test (AUDIT) for alcohol misuse.

There is some evidence to show that one-to-one professional psychological

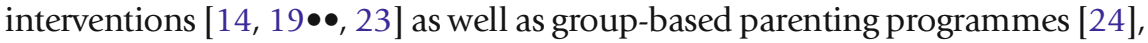

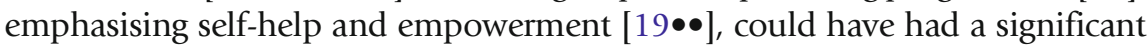
impact on the multiple risk factors (IPV, depression, alcohol misuse), that affected Lucy's mother's mental health and wellbeing.

- Accidents

Lucy's presentations to the local hospital's emergency department (ED) with accidents were also 'missed opportunities' that may have provided a trigger for further intervention by a health visitor such as one-to-one, home-centred parenting safety interventions [25•]. It is interesting to note that as well as improving injury rates, these interventions may have additional effects on parental mental health and children's behaviour. Multifaceted interventions remain useful not only in the immediate period post birth but also in the early years.

Early notification by the emergency department to the health visitor would also enable her to explore wider safety issues for Lucy as recommended in the 'Healthy Child Programme' [26].

The Common Assessment Framework (CAF) was designed as a holistic tool for multiple disciplines of professionals (including social workers and health visitors) to identify such safety issues. Whilst an admirable concept, there are concerns that it is bulky, requires descriptive demand, is often not filled completely and its structure prohibits appreciation of complexity [27].

The conclusions from the evidence of Lucy's case so far seem clear-she and her family are victims of the multiple risks and effects of social adversity. As paediatricians and advocates for children, is it not our moral duty to identify risk and minimise its impact on Lucy's life chances [28•, 29]-if we want Lucy to grow up to be a fully contributing member of society? So then, how is it possible to do this, practically?

In the antenatal and preschool years, there needs to be opportunities for trained professionals to use validated tools to screen for specific risks such as IPV, depression, alcohol misuse and accidents, or holistic tools that identify multiple safety issues. These tools need to be practical and easy to use, connecting 'screening' to intervention services. Screening and interventions work best in a patient-centred, multifaceted fashion, acknowledging the complexity within the system and the individual. This requires sufficient capacity, competencies and confidence in the children's workforce-a significant challenge in the current climate of austerity. 
- Contact with social services

Lucy's contact with social services was sporadic. There were good times (as witnessed by the second social worker) and difficult times (later, when Lucy had to care for her mother with little support). This highlights the challenge of assessing children in a snapshot of time. She 'ticked the boxes' at one point in time and therefore did not meet the threshold criteria for intervention. However, when she did require intervention, later on, it was no longer available to her. The fluctuating nature of Lucy's circumstances required a dynamic and responsive service. Laming raised this in his extensive review of child protection practice [30]. He was concerned that thresholds are a way of restricting resources and have no statutory basis-they are often set too high and cases can be missed.

- Dealing with did not attends (DNAs)

Another 'cry for help' came at age 14, when Lucy was referred to mental health services (CAMHS) by her general practitioner, for low mood. Unfortunately, due to her non-attendance, she was discharged from the system. DNAs can signal a safety concern and there needs to be systems in place to evaluate possible safeguarding concerns [31]. This could involve re-connecting with the patient and their general practitioner. It is also worth considering the reframing of non-attendance as 'Was Not Brought'. This provides a useful shift in understanding, from the child safety perspective, in acknowledging the parental role. Had this been considered, Lucy may have benefited from access to initial psychological therapy and/or medication for depression and she might have been monitored for recurrence of depressive symptoms [32].

- Age 18 transition

Once multiple issues were identified at age 17, as Lucy 'transitioned' to adult services, nothing much was done for her. Should transition be based on the specific age of the child or rather their needs and levels of maturity and understanding? The Chief Medical Officer's (CMO) report into Public Mental Health in the UK identified the 'life course' nature of mental health disorders [33] which often originate in childhood and further develop in adulthood. However, it also noted that the current transition between children and adult services is poor, as in Lucy's example. Therefore, both the $\mathrm{CMO}$ report [33] and the later "The Future in Mind' policy paper [34] have suggested that extending CAMHS until the age of 25 years would be a useful option. If this had occurred, Lucy may not have fallen through the gap between services.

Three key themes emerge when re-examining Lucy's story and considering interventions: relationships between professionals, roadmaps (use of protocols and guidance), and recognising the child at the centre (see Table 1). 


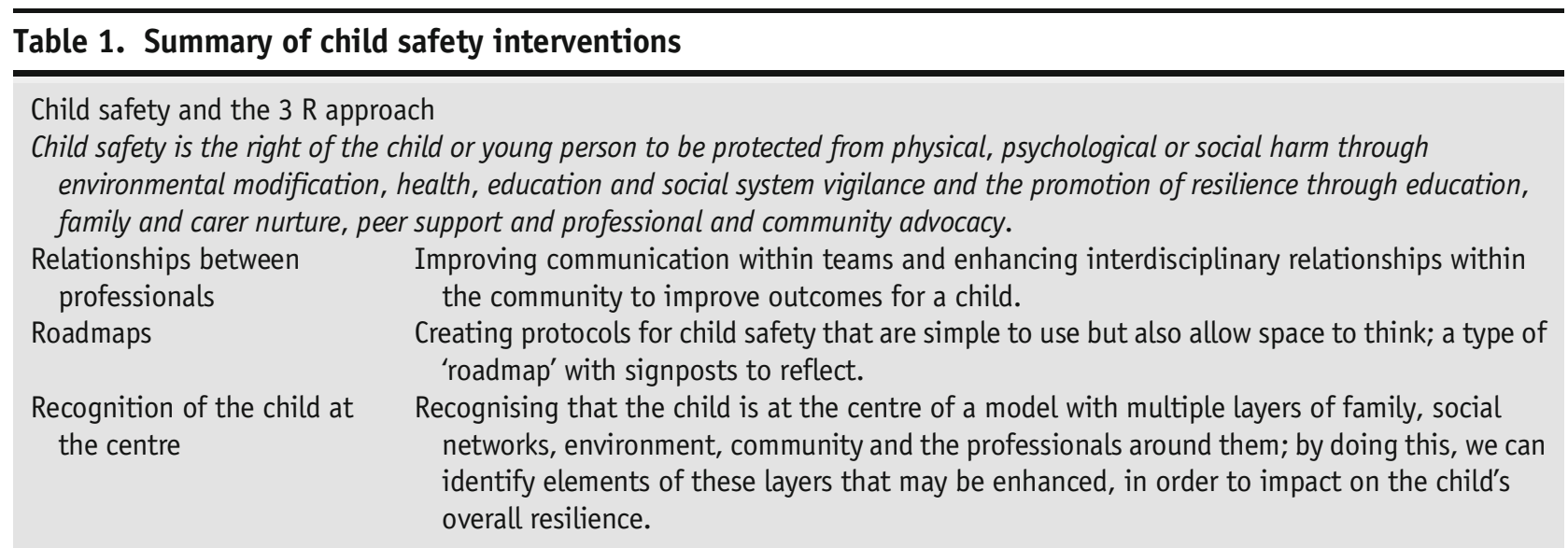

- Team communication

Systems appear to have failed Lucy, in particular, communication between professionals. Sequential enquiries of individual child protection failures have concluded with very similar, if not, identical recommendations to improve inter-professional communication emphasising the need for appropriate and timely exchange of information between agencies [30]. A 'no blame culture' or 'learning organisation' can contribute significantly to system safety outcomes [35] however, only if individuals are truly functioning as a team.

A central issue is building sustainable and respectful relationships between staff, from multiple disciplines, working across locations and each using professional judgement in the context of formal procedures. Such relationships can be enhanced by inter-professional training $[36,37]$ and the use of joint information and communication systems.

The Laming report [30] recommended setting up a national children's database to communicate between teams. 'Contact point' in England was one such attempt to have a shared database but this initiative was withdrawn. Recent information sharing guidance [38] suggests we should facilitate exchange of information which might impact on child safety. Looking at Lucy's case, had details been shared with the antenatal team, passed on to the health visitor and later re-enforced when social services had been involved-would Lucy have ended up in the place she was, at age 18 ?

- The paediatrician in the community

With regard to the interagency relationships, it is useful to look at the role of the paediatrician working within the community. In the UK, specific subspeciality 'community paediatricians' exist and have a unique opportunity to develop and foster interagency relationships in schools and communities; with a holistic vision and a preventative orientation [39]. In the USA, the American Academy of Pediatrics (AAP) has emphasised the importance of the paediatrician's role to advocate for children, collaborate with other professionals and provide health care with the context of a community [40]. It would appear that Lucy may have 
benefited from paediatrician with a community orientation, to advocate and to make the connections. However, the capacity for paediatricians to take on this role is challenging. In the $\mathrm{UK}$, many community paediatrician posts remain unfilled and it is becoming an increasingly subspecialist or even tertiary speciality, leaving little time for advocacy and interagency connections [41]. In the USA, paediatricians are spending less time on community-orientated activities, and if they do so, they are more likely to do this in a voluntary capacity [42]. At a time of austerity, we have a paradox, with increased numbers of vulnerable children yet, what appears to be, a devaluation of advocacy and community connections within our profession. If funding models can change to reward community work [43] and we can move towards integrated care models that include community agencies [44], it is possible that children like Lucy will be less likely to slip through the net. An example is the Canadian 'RICHER' initiative [45] which shows that a community-centred interdisciplinary health care model can benefit the most vulnerable children and families.

- Protocols and check lists

Reflecting on Lucy's story, we can pinpoint events where protocols may have been of use. However, the issue does not appear to be lack of protocols, as there is plenty of guidance available $[1,11,14,19 \bullet \bullet, 23,25 \bullet, 30,46-49]$. It is perhaps the way that the 'guidance' is used.

Bulky documents are often unfriendly and do not acknowledge 'human factors'. A large NICE or AAP guideline with multiple sections starkly contrasts with an airline cockpit protocol-a one-sided card with bullet point checks on it, basics that must be covered to prevent catastrophic error. In addition, the check list can be enhanced [50] with simulation training to review required crisis thinking and the impact of 'capabilities and limitations' of the crew (human factors).

For Lucy, an example of a check list, that may have been used in the context of her headaches is 'HeadSmart' [51] which gives key points to rule out in a headache history to make sure the child is 'safe' and to avoid missed diagnosis of space occupying lesions. Lucy was 'safe' from a medical point of view because she had been risk stratified for a tumour, but was she safe using our definition of promoting wellbeing? Herein lies the inherent fault of checklists and protocols-they are dependent on the user's ability to not only follow the protocol but also to think beyond its scope and look at the child holistically. It is the human factor, the user themselves, that enhances the efficacy of a protocol.

- Space to think

Bringing human factors training and awareness into medical school is vital. We can bridge the gap between practical and theoretical training through simulation and teaching doctors to understand their own thinking bias or blind spots. Acknowledging how changes in environment, time of day, mood and situation impacts directly on their ability to think, gives them greater insight into their everyday practice and improves decision-making $[52,53]$ 
Disseminating check lists, based on evidence, can improve safety outcomes-the 'Back2Sleep' campaign for infants is a good example of a Public Health initiative that used this principle [54]. However, evidence-based medicine calls for educated judicious use of this information, within the context of a patient-centred approach [55]. So how can this be achieved practically? A simple compromise would be putting in place what we call a 'think-box' within a protocol, a type of 'roadmap' with signposts, that allows space for reflection-this focuses on the subtleties of judgement and decision-making in a whole system, not purely on the biological pathophysiological aspects of disease.

\section{Recognition of the child at the centre}

- Holistic methods of assessment

With the benefit of hindsight, Lucy's contact with paediatrics for headache appears to have been a lost opportunity to examine her life from a biopsychosocial model, perhaps using a tool, such as the one in Fig. 1.

It is known as the Bioecogram though equally, other holistic tools exist. The usefulness of this tool is that it can be filled in quickly during clinic and gives a pictorial overview of the complexities of Lucy's life. It is based on the Mandala of Health (Fig. 2).

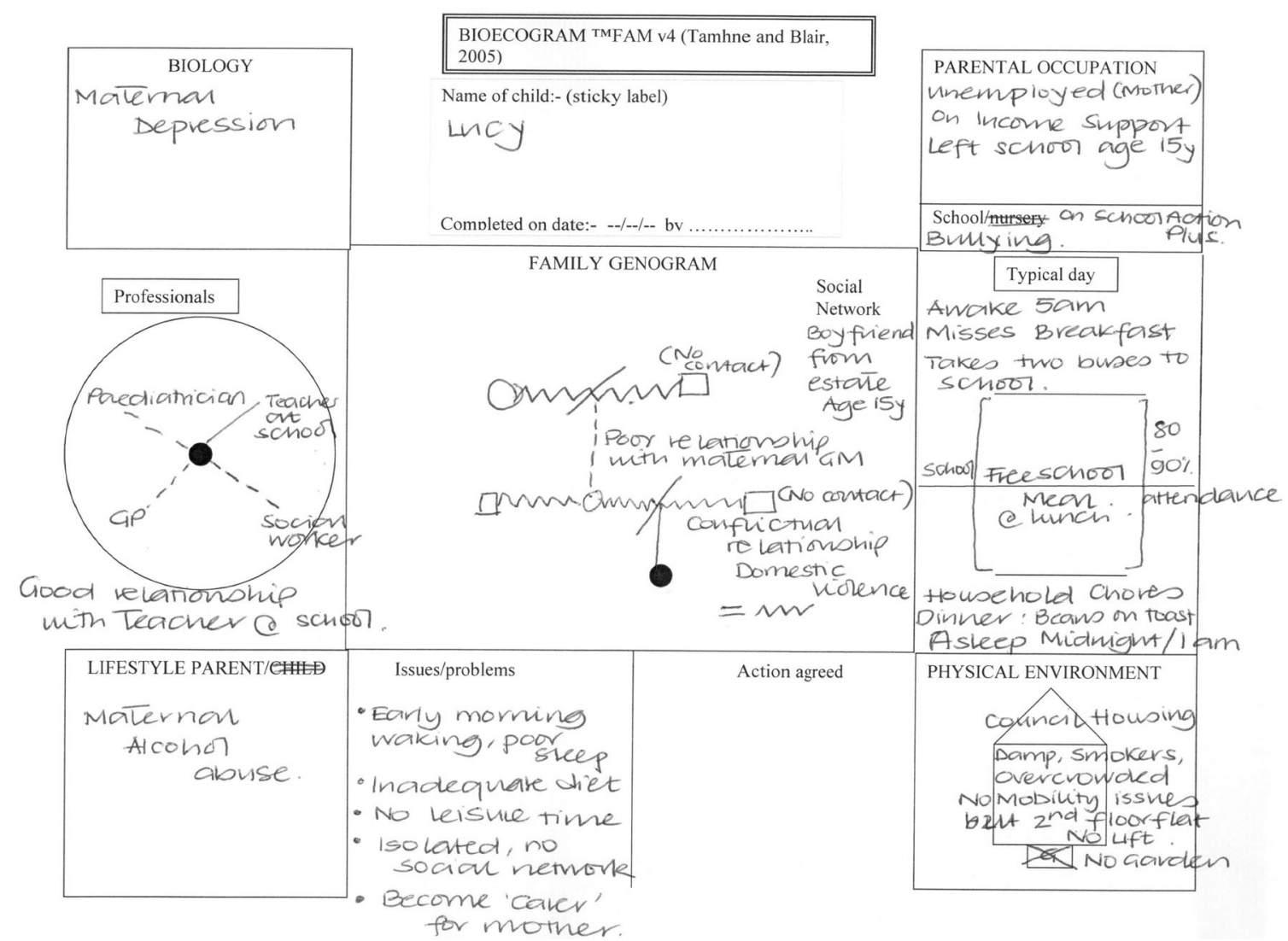

Fig. 1. Lucy's Bioecogram. Adapted from [64]. 


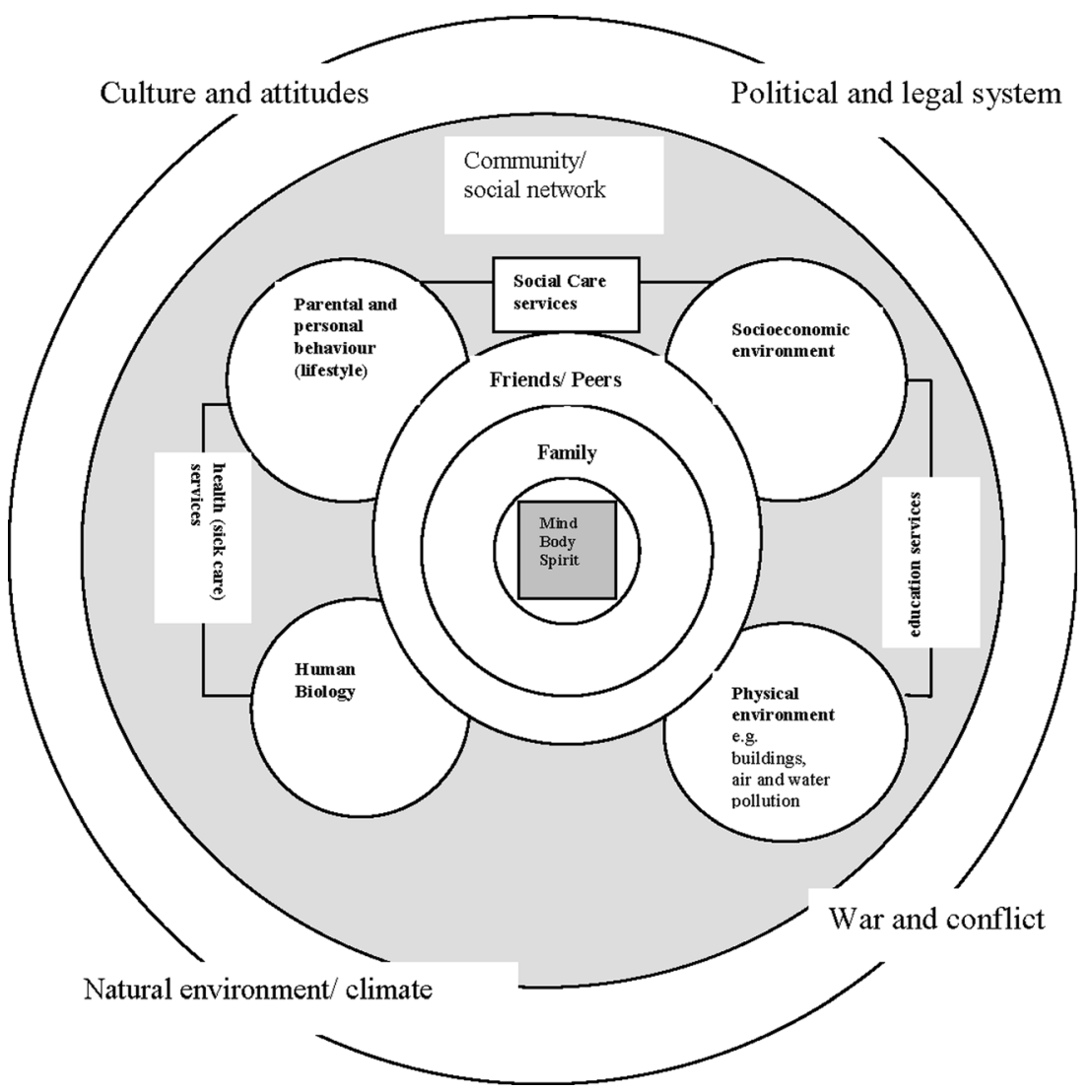

Fig. 2. Mandala of Health [65].

We can see multiple safety issues have been identified-maternal alcohol abuse, depression, and domestic violence, struggling at school, poor sleep and diet. In particular, we realise Lucy became the carer for her mother, doing all the household chores with no leisure time for herself. It would have given us a different perspective on her 'tension' headaches.

Using a lens such as the Mandala of Child Health (Fig. 2)-a visible manifestation of protective and risk factors becomes apparent and is clearly structured with the child at the centre. Initially concentrating on the child and their place within the family, adding insulating layers of neighbours, social networks, physical environment and school, keeps the child within the local community and theoretically safer. This is what was lacking overall for Lucy. Within this structure comes the current focus on mental health [34], building resilience personally [7], for the parents [24] and for the community as a whole, with structured programmes on many levels [56-58]. The African proverb 'It takes a village to raise a child' springs to mind here, and we would advocate for solutions that allow there to be many layers of support, in order to prevent a child 'slipping through the net', promoting wellbeing and preventing harm. It is worth considering that though recent evidence suggests that even just one consistent and supportive adult role model can help looked after children build more resilience [58] extending the ways in which this adult can be supported and can support their child makes sense. 
- Building resilience in children, families and communities So how do we 'build resilience'? The New Economics Foundation has recently published the five ways to wellbeing [59], looking at improving resilience amongst young people: connect, be active, take notice, keep learning and give. The challenge is creating the opportunities for young people to engage in this way.

As mentioned previously, resilience may be improved in parents, through the use of multifaceted parenting programmes [23, 24, 25•], and programmes that are group-based add an element of peer support [24]. Perhaps, Lucy's own situation may have been enhanced by the chance to connect with and volunteer within the community [49]. Her involvement in a relevant community-based project with other residents may have helped build not only her own resilience but that of the wider population in a form of co-production of health.

- Resilience of the professional

The co-creation of local services by patients and professionals embraces the Berwick reports' [60] recommendations of truly patient-centred care. For professionals to be engaged in this way, it requires both the time and willingness to adapt to needs and priorities.

Let us look again at the number of times doctors interacted with Lucy: after birth, twice in her pre-school years, both a general practitioner and paediatrician reviewed for headaches, and finally for depression. What went right in the clinic at 17.5 years? Did Lucy have a holistic interaction? Perhaps here some time and space had been carved out for her to express herself and her needs.

How can we incorporate more of this thinking into other pressurised environments? To be open to our patients' priorities requires a sense that they are not in conflict with our own. When staff feel rushed, unsafe or dissatisfied, this correlates with poor care [61, 62]. Resilience, resourcefulness and feeling supported are important for clinical staff, and the culture of a department may impact on how patients are treated [60]. Hence why this is a major focus for many hospitals now, and think tanks such as The King's Fund [63] recommend moving from rigid managerial practice to dynamic leadership and medical engagement, knowing that empowering staff to feel more in control can provide better holistic and safer care for their patients $[53,63]$.

We hope that we have demonstrated with Lucy's case study that there were a number of opportunities throughout her life course to enhance her safety and wellbeing. We have seen how the use of various holistic and more focussed assessment tools has the potential to identify risk and use evidence-based interventions to prevent 'injury'. For interventions to be successful, positive relationships need to be developed between the multiple professionals around the child. The use of standardised protocols or roadmaps can improve this process if designed with the user in mind, if suitable training and support is present. Recognising the child at the centre and their own ability to deal with adverse events as well as supporting the familial, community and professional network around the child results in greater resilience and hopefully better life outcomes. 
Child Safety in the community remains a complex issue involving the interaction of multiple systems across the life course. Solutions need to acknowledge that complexity, and require further thinking and action at a local, national and international level. As such, we would encourage professionals working with children to embrace their role as advocates, because investing in children and helping them to achieve their potential pay dividends for their future and for our society.

\section{Compliance with Ethics Guidelines}

\section{Conflict of interest}

Mitch Blair, Shermina Sayani and Elizabeth Wortley declare that they have no conflict of interest.

\section{Human and Animal Rights and Informed Consent}

This article does not contain any studies with human or animal subjects performed by any of the authors.

\section{References and Recommended Reading}

Papers of particular interest, published recently, have been

highlighted as:

- Of importance

$\bullet \quad$ Of major importance

1. Kendrick D, Young B, Mason-Jones AJ, et al. Home safety education and provision of safety equipment for injury prevention. Evid-Based Child Heal A Cochrane Rev J. 2012;8:761-939.

2. Lozano R, Naghavi M, Foreman K, et al. Global and regional mortality from 235 causes of death for 20 age groups in 1990 and 2010: a systematic analysis for the Global Burden of Disease Study 2010. Lancet. 2012;380:2095-128. doi:10.1016/S0140-6736(12) 61728-0.

3. European Child Safety Alliance. http:// childsafetyeurope.org/index.html. Accessed 252015.

4. CEOP Command. http://www.ceop.police.uk/. Accessed 25 Jun 2015.

5. Department for Education (2013) Working together to safeguard children. https://www.gov.uk/government/ publications/working-together-to-safeguard-children. Accessed 3 Jul 2015.

6. Kohn LT, Corrigan JM, Donaldson MS, et al. (2000) To err is human: building a safer health system. National Academies Press.

7. Rutter M. Resilience as a dynamic concept. Dev Psychopathol. 2012;24:335-44. doi:10.1017/ S0954579412000028.

8. Greenhalgh T, Hurwitz B. Why study narrative? West J Med. 1999; 170:367-9.

9. Cass H (2006) The NHS experience: the "Snakes and Ladders" guide for patients and professionals. Psychology Press.
10. Kuh D, Ben-Shlomo Y (1997) A life course approach to chronic disease epidemiology, First. Oxford University Press.

11. National Institute for Health and Care Excellence (2014) Domestic violence and abuse: how health services, social care and the organisations work with can respond effectively. http://www.nice.org.uk/guidance/ ph50. Accessed 3 Jul 2015.

12. Shah PS, Shah J. Maternal exposure to domestic violence and pregnancy and birth outcomes: a systematic review and meta-analyses. J Womens Health (Larchmt). 2010;19:2017-31.

13. Rodriguez E, Lasch KE, Chandra P, Lee J. The relation of family violence, employment status, welfare benefits, and alcohol drinking in the United States. West J Med. 2001;174:317-23.

14. Jahanfar S, Howard LM, Medley N (2014) Interventions for preventing or reducing domestic violence against pregnant women. Cochrane Libr.

15. Winnicott DW. Collected papers: through paediatrics to psycho-analysis. London: Tavistock Publications; 1958.

16. Bowlby J. Attachment: attachment and loss (volume 1). London: Hogarth; 1971.

17. Ainsworth MS. Infant-mother attachment. Am Psychol. 1979;34:932.

18. Cummings EM, Davies PT. Maternal depression and child development. J Child Psychol Psychiatry. 1994;35:73-112. 
19.• National Institute for Health and Care Excellence (2014) Antenatal and postnatal mental health: clinical management and service guidance. http://www.nice. org.uk/guidance/cg192/chapter/1-recommendations. Accessed 3 Jul 2015.

A detailed systematic review of screening opportunities and interventions in the antenatal and postnatal period. These may identify and manage the risk factors in mothers preventing subsequent safety issues for their children.

20. Cox JL, Holden JM, Sagovsky R. Detection of postnatal depression. Development of the 10-item Edinburgh Postnatal Depression Scale. Br J Psychiatry. 1987; 150:782-6.

21. Shakespeare J. Health visitor screening for PND using the EPDS: a process study. Community Pract. 2002;75:381.

22. Anda RF, Whitfield CL, Felitti VJ, et al. Adverse childhood experiences, alcoholic parents, and later risk of alcoholism and depression. Psychiatr Serv. 2002;53:1001-9.

23. Dennis CL, Creedy D (2013) Psychosocial and psychological interventions for preventing postpartum depression. Cochrane Database Syst Rev CD001134. doi:10.1002/14651858.CD001134.pub2

24. Barlow J, Smailagic N, Huband N, et al. (2014) Groupbased parent training programmes for improving parental psychosocial health. Cochrane Database Syst Rev

25. Kendrick D, Mulvaney CA, Ye L, et al. (2013) Parenting interventions for the prevention of unintentional injuries in childhood. Cochrane database Syst Rev ho.

A detailed review of the parenting interventions (parenting programmes) that can prevent childhood injury, in particular, one to one interventions, delivered in the home by a trained professional, show promise.

26. Department of Health (2009) Healthy child programme: pregnancy and the first 5 years of life. https:// www.gov.uk/government/publications/healthy-childprogramme-pregnancy-and-the-first-5-years-of-life. Accessed 3 Jul 2015.

27. White S, Hall C, Peckover S. The descriptive tyranny of the common assessment framework: technologies of categorization and professional practice in child welfare. Br J Soc Work. 2009;39:1197-217.

28. Davies SC (2013) CMO's annual report 2012: Our children deserve better: CMO's summary as a web page. https://www.gov.uk/government/publications/chiefmedical-officers-annual-report-2012-our-childrendeserve-better-prevention-pays/cmos-annual-report2012-our-children-deserve-better-cmos-summary-as-aweb-page. Accessed 23 Jun 2015.

An in depth view of child health and wellbeing as part of the child medical officers 'State of the Nation' report. It gives the moral and economic argument for why investing in children is important for them as individuals and for our whole society.

29. Marmot M, Bell R (2010) Fair society, healthy lives. Public Health.

30. Laming WH (2009) The protection of children in England. A progress report.
31. Arai L, Stephenson T, Roberts H. The unseen child and safeguarding: "did not attend" guidelines in the NHS. Arch Dis Child. 2015;100:517-20.

32. Wolpert M, Fuggle P, Cottrell D, et al. (2006) Drawing on the evidence: advice for mental health professionals working with children and adolescents-2nd ed.

33. Ford T, Mitrofan O, Wolpert M (2013) Annual report of the chief medical officer 2014, public mental health priorities: investing in the evidence: Chapter 6: life course: children and young people's mental health.

34. Department of Health (2015) Future in mind.

35. Wu AW. Medical error: the second victim. BMJ Br Med J. 2000;320:726-7.

36. Polnay J, Blair M. A model programme for busy learners. Child Abus Rev. 1999;8:284-8.

37. Bannon MJ, Carter YH. Paediatricians and child protection: the need for effective education and training. Arch Dis Child. 2003;88:560-3.

38. Department of Health (2012) Information requirements for child health information systems.

39. Curtis E, Waterston T. Community paediatrics and change. Arch Dis Child. 2002;86:227-9. doi:10.1136/ adc.86.4.227.

40. Rushton FE. The pediatrician's role in community pediatrics. Pediatrics. 2005;115:1092-4. doi:10.1542/ peds.2004-2680.

41. Mather M. Community paediatrics in crisis. Arch Dis Child. 2004;89:697-9. doi:10.1136/adc.2003.038950.

42. Minkovitz CS, Grason H, Solomon BS, et al. Pediatricians' involvement in community child health from 2004 to 2010. Pediatrics. 2013;132:997-1005. doi:10. 1542/peds.2013-1917.

43. Chamberlain LJ, Kaczorowski JM. "You Get What You Pay For": resources for training and practice in community pediatrics matter. Pediatrics. 2014;134:173-5. doi:10.1542/peds.2014-1130.

44. Montgomery-Taylor S, Watson M, Klaber B (2015) Child health-leading the way in integrated care. J R Soc Med:1-5. doi:10.1177/0141076815588315

45. Wong ST, Lynam MJ, Khan KB, et al. The social paediatrics initiative: a RICHER model of primary health care for at risk children and their families. BMC Pediatr. 2012;12:158. doi:10.1186/1471-2431-12-158.

46. National Institute for Health and Care Excellence (2012) Obesity: working with local communities. NICE public health guidance.

47. Sahuquillo J, Arikan F (1996) Cochrane database of systematic reviews. Cochrane Database Syst Rev CD003983.

48. Smailagic N, Huband N, Roloff V, Bennett C. Groupbased parent training programmes for improving parental psychosocial health. Cochrane Libr. 2012. doi:10.1002/14651858.CD002020.pub3.

49. Jenkinson $\mathrm{CE}$, Dickens $\mathrm{AP}$, Jones $\mathrm{K}$, et al. Is volunteering a public health intervention? A systematic review and meta-analysis of the health and survival of volunteers. BMC Public Health. 2013;13:773.

50. Degani A, Wiener EL. Cockpit checklists-concepts, design, and use. Hum Factors. 1993;35:28-43. 
51. Headsmart Head Smart. http://www.headsmart.org. uk/. Accessed 25 Jun 2015

52. Groopman J (2008) How doctors think. Mariner books.

53. Royal College of Paediatrics and Child Health (2015) S.A.F.E. programme-situation awareness for everyone. http://www.rcpch.ac.uk/safe. Accessed 13 Jul 2015

54. Meadows-Oliver M, Hendrie J. Expanded back to sleep guidelines. Pediatr Nurs. 2013;39:40-9.

55. Sackett DL, Rosenberg WMC, Gray JAM, et al. Evidence based medicine: what it is and what it isn't. Br Med J. 1996;312:71-2.

56. Allen M (2014) Building children and young people's resilience in schools.

57. NHS England (2015) Building the NHS of the five year forward view-NHS England Business plan 2015/16. http://www.england.nhs.uk/2015/03/27/businessplan-2015-16/. Accessed 13 Jul 2015

58. Yeadon A, Share A. G57(P) fostering resilience: the promotion of resilience in young people who are looked after. Arch Dis Child. 2015;100:A24-4.
59. NEF five ways to wellbeing. http://www. fivewaystowellbeing.org/. Accessed 13 Jul 2015

60. Department of Health (2013) Berwick review into patient safety.

61. Francis R (2013) Report of the Mid Staffordshire NHS Foundation Trust public inquiry. http://webarchive. nationalarchives.gov.uk/20150407084003/http:// www.midstaffspublicinquiry.com/report. Accessed 2 Sept 2015.

62. Keogh B (2013) Review into the quality of care and treatment provided by 14 hospital trusts in England: overview report. http://www.nhs.uk/nhsengland/ bruce-keogh-review/documents/outcomes/keoghreview-final-report.pdf. Accessed 13 Jul 2015

63. West MA, Dawson JF (2012) Employee engagement and NHS performance. King's Fund 1-23.

64. Blair M, Tamhne R (2004). The Bioecogram: a novel assessment tool for the enhancement of family paediatric practice and child-centred care. Pediatr. Acad. Soc. Annu. Sci. Meet. San Fr.

65. Blair M (2010) Child public health. Oxford University Press 